\author{
Military Technical College \\ Kobry El-Kobbah, \\ Cairo, Egypt
}

$14^{\text {th }}$ International Conference on Applied Mechanics and Mechanical Engineering.

\title{
Gear Wear Accelerated testing Device with Reverse motion - Design and Application -
}

\author{
By \\ Milosav Ognjanovic* Ana Pavlovic ${ }^{* *} \quad$ Snezana Ciric-Kostic***
}

\begin{abstract}
:
Gear wear testing is an extensive laboratory process in conditions similar to service ones. To apply the obtained results for the design of the new gearing structures, it is necessary to identify an additional indicator: the wear probability. In this case reverse gear motion is an additional condition which is necessary to simulate along the laboratory testing. Design, including 3D model of testing device structure for the purpose of plastic gear wear testing, is presented and analyzed. The application for wear probability testing of gears with reverse motion, together with experimental data processing, is presented. The application of those results in design process of the new gear structures is discussed. Gear system for air flow and combustion control in the car engines is used as an example for testing and design. Plastic gears transform control motion and load by resistance torque of the spring for reverse motion. Service life and reliability of this system, including its design using reliability as design constraint, needs experimental data obtained by designed testing device.
\end{abstract}

\section{Keywords:}

Gears, Wear, Failure probability, Testing device 
* University of Belgrade, Faculty of Mechanical Engineering, Serbia

** University of Bologna, DIEM-Faculty of Engineering, Italy

*** University of Kragujevac, Faculty of Mechanical Engineering Kraljevo, Serbia

\section{Introduction:}

Testing rigs for rotating systems, including gears and gear transmission units, in general, use the two basic principles: open system and closed system of power circulation i.e. back-toback system. The first, open system is adaptive for the testing of various designs but in a shorter duration of testing. For the long time testing, until the failure of the tested sample, the closed system is much more efficient and economic. The same power (load) circulates in the system. Outside (input) energy is spent for friction and similar resistances are overcome only. The gear system for air flow control in car engines is not really suitable for application of this system, the system design is specific and motion is reverse. This is the reason why it was necessary to develop the specific testing device which will obtain some additional advantages for that testing.

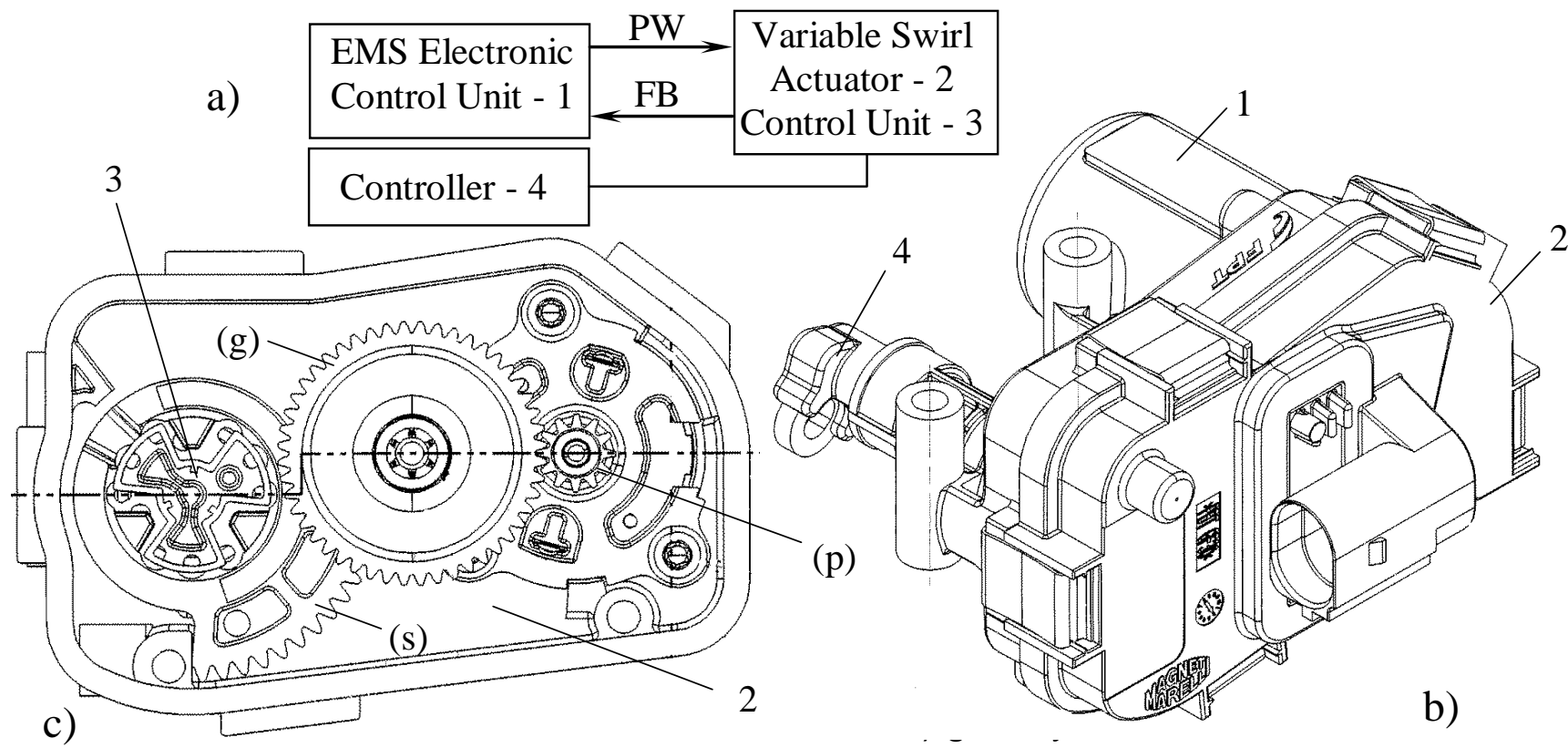

Figure (1): The unit for air flow control

In Figure 1 the unit for air flow control in the car engines is presented. Electronically controlled motor 1 produces the power PW for air flow control. This power is transformed by the gear unit 2. The gear unit motion is controlled by sensors 3 directly connected by the shaft with flow valve controller 4 . The spring for reverse motion is settled behind the sensors' disc 3 around the connecting shaft. Gear unit 2 consists of the two gear pairs. The first pair presents the metal pinion (p) in conjugation with the gear $(g)$. The gear $(\mathrm{g})$ in the same piece has the two toothed rings. The small ring which is behind the large one is in conjugation with the toothed segment (s). The segment is in one material piece with control unit 3 and connecting shaft with rotation of 90 degrees. 


\section{Testing device design:}

For the testing of the gear system presented in Fig. 1 it is necessary to provide a set of conditions which can keep service conditions in real service life and accelerate the testing process in order to obtain teeth wear failure probability with minimal duration of the testing. In this regard, it is necessary by testing device to provide as follows:

1. The motion has to be reverse and fulfill a limited range of gear segment rotation. The testing device has to provide increase of the motion speed in order to accelerate the testing process.

2. The system has to provide change of the gear load and also load increase in order to accelerate the testing process.

3. The temperature of the gear flanks has to be similar to the temperature in service conditions.

4. The number of the testing samples under the same conditions has to provide wear probability calculation i.e. minimum eight tested samples.

5. Easy handling (load and speed variation) and wear process monitoring.

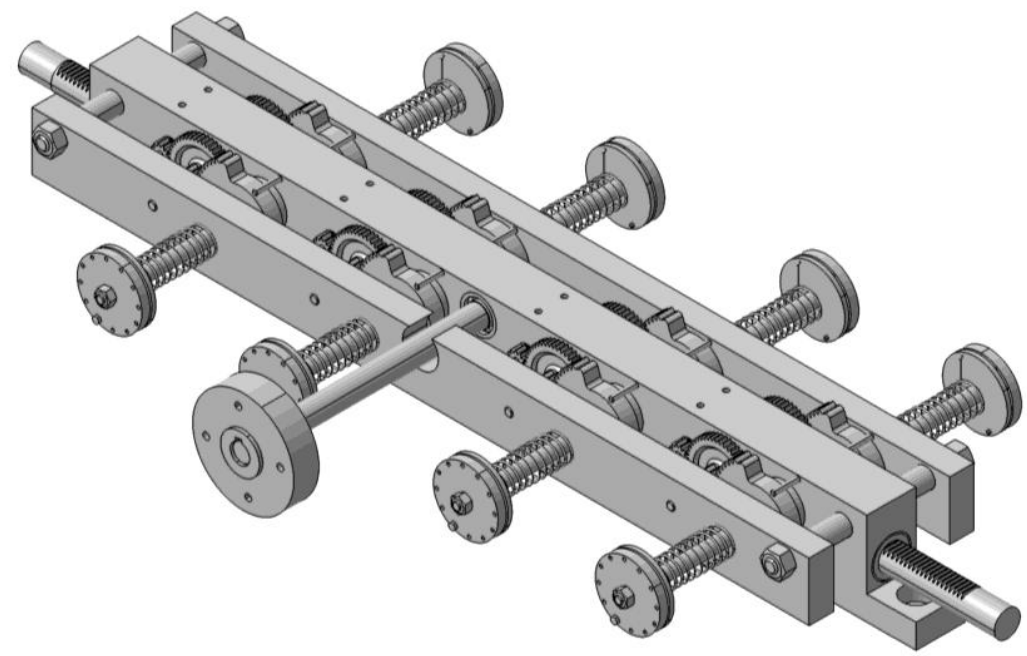

Figure (2): Central part of the testing device 


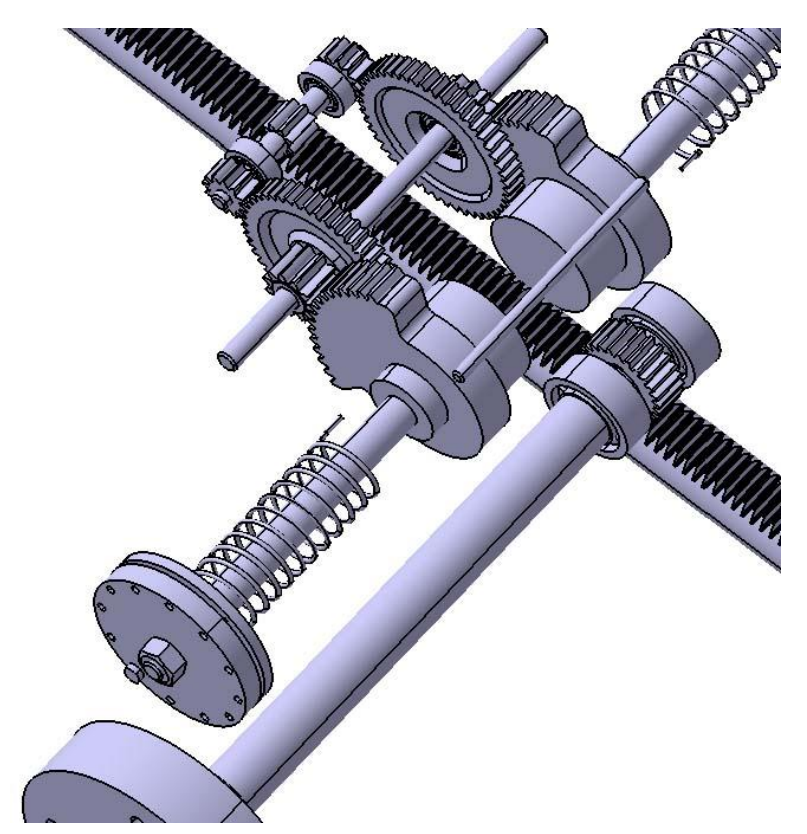

Figure (3): Assembly of gears with load and motion components

In Figures 2 and 3 the central part of the testing device is presented. Input coupling 1 is connected with electronically controlled driving system which produces reverse rotation with programmed number of revolutions in one and another direction and with programmed speed. The input shaft is conjugated by the gear 2 (Fig.3) with the toothed rack 3 . The rack is supported in the two linear rolling bearings. The rack is also conjugated with the four gears 4 (Fig.3), which drives left and right tested samples i.e. tested gear assembly. By motion in both directions the toothed bar produces oscillator motion of the shaft 5 . The shaft is loaded by torque caused by spring 6 . Preloading of the spring is provided by the two discs 7 and 8 . The disc 7 is connected with the shaft 5 and the disc 8 with the spring 6 . Both discs have the holes on their peripheries. By the bolt 9 the disc 8 is connected with the disc 7 . By the position variation of the disc 8 , the torque caused by the spring, acting on the shaft, varies. This is the way to vary and choose the necessary resistance torque before testing. Oscillator motion of the shaft with the toothed segment changes the spring deformation and resistant torque in a relatively short range. The shafts are supported in the plates 10 (Fig. 2) which are also supports for angular deformations of springs.

\section{Testing device application:}

The presented testing device is designed for the wear testing of the plastic gears with reverse motion. The device is especially adapted for testing of the gearing mechanism in engine air flow controller. These are the gears of small dimensions. The first pair (p-g) (Fig.1) is with the module of $0.8 \mathrm{~mm}$ and the centre distance of $25.6 \mathrm{~mm}$ (width $5 \mathrm{~mm}$ ), and the second pair (with the gear segment - s) has the module of $1 \mathrm{~mm}$ and centre distance of $34.5 \mathrm{~mm}$ (width $7 \mathrm{~mm}$ ). The dimensions are adapted to load and stress distributions in order to obtain optimal design solution with equal wear reliability. The testing of plastic gear wear can be carried out to fulfill the following objectives.

- To identify the gear and place where failure begins i.e. the weakest spot in the gear set assembly. 
- To identify gear wear probability for the service conditions. Failure (wear) probability for service conditions presents in itself the elementary reliability for a certain failure or for a certain machine part.

- To identify the range of failure (wear) probability distribution. The range of probability distribution is used as a function of failure probability distribution for various stress levels or for various stress cycles numbers. In combination with various service conditions (probability of service parameters) the failure probability obtains reliability calculation.

The testing device obtains parallel testing of eight gear sets with the same load (torque at the shaft) and with the same speed of motion. The stress cycles number to the failure can be measured and calculated. The wear failure does not arise after the same stress cycles number. The failed gear sets can be removed or replaced with the new gear sets and test can be continued until to failure of the next gear set. Parallel testing of the eight samples provide the possibility to obtain the set of results, which is sufficient for the probability or reliability calculation. This is one way for testing the process of acceleration. The possibility for the motion speed increase compared to real service conditions can also significantly accelerate the testing process. Increase of load (shaft torque) can accelerate the testing process but can significantly change the conditions in the teeth mesh. Increase of temperature level with the speed or load increase can be prevented by tested samples cooling. If it is necessary, when service temperature is higher, the tested samples can be heated. For the tested samples heating or cooling, the tested device has to be covered and closed in the housing for this purpose.

\section{Gear wear failure probability identification:}

At the beginning of gear teeth wear probability range distribution, it is necessary to make a few decisions. Firstly, in the range of finite life it is necessary to choose the two stress levels, so as to identify this range bounded by inclined strict lines (Fig. 4). The distance between these stresses levels has to be large enough in order to obtain a more precise angle of line inclination. Higher level $\sigma_{H 1}$ has to be caused by the load which can cause teeth fracture by bending stress. Lower stress level $\sigma_{\mathrm{H} 2}$ has to be high enough to avoid the range of failure distribution in infinite fatigue life range. The number of gear sets, which it is necessary to test at each of the two stress levels, is eight and defined by the testing device capacity. It corresponds to the minimal statistical set for wear probability calculation. Independent variable $N$ is the number of gear revolutions (teeth stress cycles number) until failure occurs. Also, in the case of actual gear set testing, the number of the control shaft 5 (Fig.3) oscillations can be used as the number of cycles. To identify the function of failure probability distribution $P_{F}\left(\sigma_{H}\right)$ in the infinite wear life, it is necessary to choose the stress (or control shaft oscillation) cycles number $N_{D V}$ in this range (Fig.4). The samples can fail prior to this limit or not fail until $N_{D V}$. In this range, the testing is carried out for a few levels of the stress $\square_{H}$. For each of them, the testing set of gear pairs is also minimum the eight.

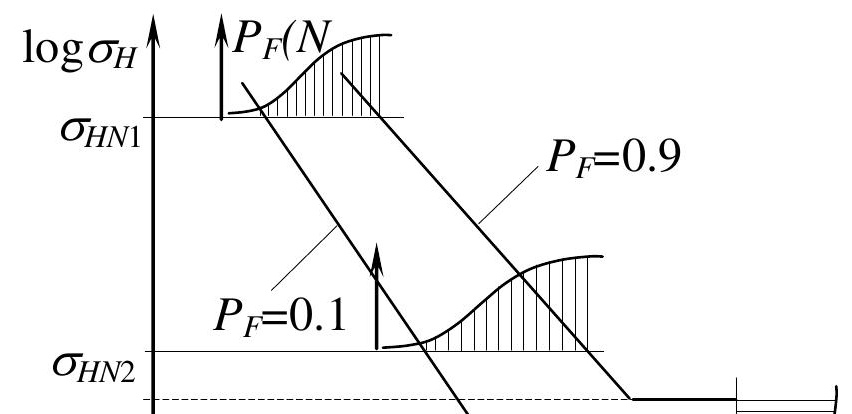


Figure (4): Gear wear probability testing

The tested results processing provides parameters of Weibull's function for wear probability distribution. The first step is calculation of empirical failure probability for every stress level $\sigma_{H N 1} \ldots \sigma_{H N 8}$. In the range of finite life for $\sigma_{H N 1}$ and $\sigma_{H N 2}$ probability for a certain stress level is calculated as

$$
P_{F}(N)=\frac{z_{i}(N)}{z_{\Sigma}+1}
$$

where $z_{i}(N)$ is the number of tested samples failed with the stress cycles number less or equal to $N$. The total number of tested samples is $z_{\square}$. For a certain stress level the calculation is carried out for $z_{\Sigma}$ failed (wear) samples, and each of them failed after different cycles number $N$. The calculated values $P_{F}(N)$ are used for Waibull's function of probability distribution i.e. parameters of this function. In the range of infinite life for the stress levels $\sigma_{H N 3} \ldots \sigma_{H N 8}$ the failure probability is calculated independently. For a certain stress level $\sigma_{H N i}$ failure probability is

$$
P_{F}\left(\sigma_{H N i}\right)=\frac{z_{i}\left(\sigma_{H N i}\right)}{z_{\Sigma}+1}
$$

$Z_{i}\left(\sigma_{H N_{i}}\right)$ is the number of samples failed after the cycles number is less or equal to $N_{D V}$. The calculated results for all stress levels $\sigma_{H N 3} \ldots \sigma_{H N B}$ are used for Weibull's function of probability distribution (function parameters) for stress cycles number $N_{D V}$.

When all of the three failure probability functions (Fig.4) are obtained (the two functions $P_{F}(N)$ and function $P_{F}\left(\sigma_{H}\right)$ ), it is possible to complete the total area of failure probability distribution bounded by lines for $P_{F=0.1}$ and $P_{F=0.9}$. Using this area of probability distribution for every stress level or for every stress cycles number, it is possible to define Waibull's function, necessary for unreliability calculation.

\section{Conclusions:}


The paper presents a new design of testing device for specific testing process of specific gear set produced of the plastic of specific characteristics. The device provides the possibility for accelerated reliability testing of the presented gear set for air flow control in the car engines. Also, the device provides the possibility for wear failure probability distribution which can be used for reliability definition in various service conditions. The set of application possibilities are presented. Additionally, the process of testing is presented. Also, the process of failure probability calculation and range of failure probability definition is discussed. Parallel testing of the eight samples (gear sets) significantly accelerate the testing process. Also, by increase of the speed of motion and by increase of the gear set load testing acceleration can be additionally accelerated. By heating or cooling of the testing samples, the application of presented device gets broader. 


\section{References:}

[1] A. Flodin, Wear of spur and helical gears, Doctoral Thesis, Department of Machine Design, Royal Institute of Technology, S-100 44 Stockholm, 2000.

[2] A. Flodin, S. Andersson, Simulation of mild wear in helical gears, Wear 241, 2000, 123-128.

[3] A. Flodin, S. Andersson, A simplified model for wear prediction in helical gears, Wear 249 (2001) 285-292.

[4] J. Rech, M.A. Djouadi, J. Picot, Wear resistance of coatings in high speed gear hobbing, Wear 250 (2001) 45-53.

[5] N.A. Wright, S.N. Kukureka, Wear testing and measurement techniques for polymer composite gears, Wear 251 (2001) 1567-1578.

[6] J. Brauer, S. Andersson, Simulation of wear in gears with flank interference - a mixed FE and analytical approach, Wear 254 (2003) 1216-1232.

[7] M. Kurokawa, Y. Uchiyama, T. Iwai, S. Nagai, Performance of plastic gear made of carbon fiber reinforced polyamide 12, Wear 254 (2003) 468-473.

[8] K. Aslantas, S. Tasgetiren, A study of spur gear pitting formation and life prediction, Wear 257 (2004) 1167-1175.

[9] E. Brinksmeier, A. Giwerzew, Hard gear finishing viewed as a process of abrasive wear, Wear 258 (2005) 62-69.

[10] U.S. Santiago, D.J. Weale, A. Wood, R. Weidig, Design and Evalution of Novel Test Rig for Analysis of the Dynamics, Load Capacity and Lifetime of High-Performance Plastic Gears, Proceedings of International Conference on Gears, VDI-Berichte 1904 (2), Munich 2005, 1185-1202.

\section{Nomenclatures:}

$\sigma_{H} \ldots$. Herzian teeth flank stress.

$\sigma_{H N \ldots} .$. Teeth flank endurance.

$N$... Cycle number to the teeth flank wear (number of gear revolutions or number of control shaft oscillations.

$N_{D V . .} \quad$ Boundary of cycle number in infinite life area.

$z_{i} \ldots$ Number of failed tested samples with the cycles less or equal to bound cycles number.

$z_{\Sigma} \ldots \quad$ Total number of samples tested at certain load (stress) level

$P_{F \ldots} \quad$ Failure (wear) probability 